\title{
Microcirurgia das lesões traumáticas de nervo periférico do membro superior
}

\section{Microsurgical treatment to repair injuries of the peripheral nerves in upper limb}

\author{
Katia Torres Batista ${ }^{1}$ \\ Hugo José Araújo ${ }^{2}$
}

Trabalho realizado na Rede Sarah de Hospitais do Aparelho Locomotor, Brasília, DF, Brasil.

$$
\begin{array}{r}
\text { Artigo submetido no SGP } \\
\text { (Sistema de Gestão de } \\
\text { Publicações) da RBCP. } \\
\text { Artigo recebido: 29/7/2009 } \\
\text { Artigo aceito: } 22 / 9 / 2009
\end{array}
$$

\begin{abstract}
RESUMO
Apesar da grande quantidade de publicações e surgimento de novos dados laboratoriais e clínicos relacionados aos conceitos da regeneração dos nervos periféricos, estas lesões são ainda um dos mais desafiadores e difíceis problemas cirúrgicos reconstrutivos. O emprego da microcirurgia tem melhorado os resultados do reparo de nervo, principalmente nas lesões agudas, contudo para reduzir as sequelas é importante a compreensão dos mecanismos, princípios técnicos e de reabilitação. Os autores realizaram revisão da literatura com enfoque para o reparo neural e a reabilitação pós-operatória.
\end{abstract}

Descritores: Nervos periféricos/lesões. Doenças do sistema nervoso periférico. Eletromiografia.

\begin{abstract}
Despite the large number of publications and development of laboratory and clinical concepts related to the regeneration of peripheral nerves, these injuries remain one of the most challenging and difficult surgical reconstructive problems. The microsurgery use has improved the outcome of nerve repair, especially in acute injuries, but to get reduce the consequences it is important to understand the mechanisms, technical principles and rehabilitation. The authors review the literature about neural repair, and rehabilitation after surgery.
\end{abstract}

Keywords: Peripheral nerves/injuries. Peripheral nervous system diseases. Electromyography.

\section{INTRODUÇÃO}

O interesse pelos estudos da cirurgia de nervo periférico existe há mais de um século. Na fisiopatologia da regeneração neural, considera-se a presença de fatores neurotróficos que influenciam no crescimento axonal. Até os dias atuais prevalecem os princípios da regeneração propostos por Millesi nos anos 70, entretanto, muito se progrediu na recuperação funcional, sobretudo com os avanços em microcirurgia e reabilitação ${ }^{1,2}$.

A recuperação sensitiva completa após reparo neural ainda é rara. Depende de fatores como a extensão da lesão, a qualidade do crescimento axonal, a orientação do crescimento neural e o processo de reorganização do córtex cerebral $^{1,2}$. Lundborg ${ }^{1}$ revisou os conceitos neurocientíficos quanto ao uso de imunossupressores, fatores de crescimento, sensibilidade artificial, implantação de microchips no sistema nervoso periférico e central, e a aplicação genética nos processos de regeneração e degeneração neural. É relevante o conhecimento do mecanismo biológico dos processos na regulação, regeneração pós-traumática do nervo, da plasticidade cerebral e da reorganização funcional ${ }^{1-4}$.

Na prática, as lesões traumáticas dos nervos são comuns e comprometem a função do membro superior. Após o reparo destas lesões são necessários tempo prolongado de afastamento do trabalho, mudanças de função e, às vezes, aposentadoria ${ }^{5-10}$. As opções para o reparo mais comumente realizadas são a neurorrafia término-terminal e a enxertia de nervo ${ }^{1,2}$. A neurorrafia término-lateral é ainda controversa.

O objetivo desse artigo é realizar uma breve revisão dos

1. Médica Cirurgiã Plástica do Hospital Sarah Brasília, Brasília, DF, Brasil; Membro Titular da Sociedade Brasileira de Cirurgia Plástica (SBCP).

2. Médico Cirurgião Plástico do Hospital Sarah Brasília, Brasília, DF, Brasil; Membro Titular da Sociedade Brasileira de Cirurgia Plástica (SBCP). 
recentes conceitos no reparo e reabilitação das lesões de nervo periférico do membro superior.

\section{MÉTODO}

Iniciou-se a busca na literatura mundial nas bases de dados Medline/PubMed, LILACS e Google acadêmico. Procuraram-se artigos sobre os aspectos dos tópicos: regeneração, estudo de condução, reparo e reabilitação das lesões traumáticas de nervo periférico no membro superior. Utilizaram-se como descritores: "nervo periférico, lesões de nervos e lesão de nervo no membro superior" ("peripherical nerve, nerve injuries, upper extremity nerve injuries"). Foram recuperados 33.970 artigos relacionados ao primeiro e segundo descritores, 166 publicados no ano de 2008 e 3653 ao último descritor. Os artigos foram selecionados por ano, atualização e relevância na aplicação clínica, conforme a experiência dos autores.

\section{REGENERAÇÃO NEURAL}

Muito do entendimento do processo de regeneração neural advém de modelos animais. O sistema de nervo periférico tem considerável capacidade de regeneração. Após a lesão do nervo ocorrem alterações estruturais, degenerativas no coto proximal, distal e no espaço entre eles. A mielina é desbridada pela ação dos macrófagos, preparando o coto distal para recepção de fibras originadas do coto proximal. Durante essa fase, ocorre a multiplicação das células de Schwann, fibroblastos e aumento do colágeno. O coto proximal é o sítio de intensa atividade e multiplicação das células de Schwann, que migram formando as Bandas de Burgner ${ }^{2,3}$.

O espaço entre os cotos é preenchido por sangue contendo macrófagos. O coágulo de fibrina forma-se preenchido por capilares, fibroblastos e terminações nervosas. Os brotos de tecido axonal acompanham as células de Schwann ${ }^{2-4}$. Quando se interpõe um enxerto de nervo, este age como uma origem de células de Schwann para sustentação dos brotos axonais e condução ao coto distal. A sobrevivência, o crescimento e a distância entre as fibras depende de fatores humorais neurotróficos, neurotrópicos e do perfeito alinhamento fascicular ${ }^{3}$. Os axônios do coto proximal penetram através do sítio de reparo e progridem lentamente, até $1 \mathrm{~mm} /$ dia, principalmente nos enxertos. Este processo pode durar semanas a meses até atingir a placa motora e gerar função. A maturação das fibras e a recuperação do volume axonal com espessamento mielínico pode durar até 5-6 anos, dependendo da localização da lesão $0^{1,4}$ (Figura 1).

Para propósitos clínicos, há três caminhos básicos descritos por Sunderland ${ }^{3}$, nos quais a fibra neural responde ao trauma, à neuropraxia, à axonotmese e à neurotmese de Grau I, II, III, IV e V. Na neuropraxia (Grau I), há um

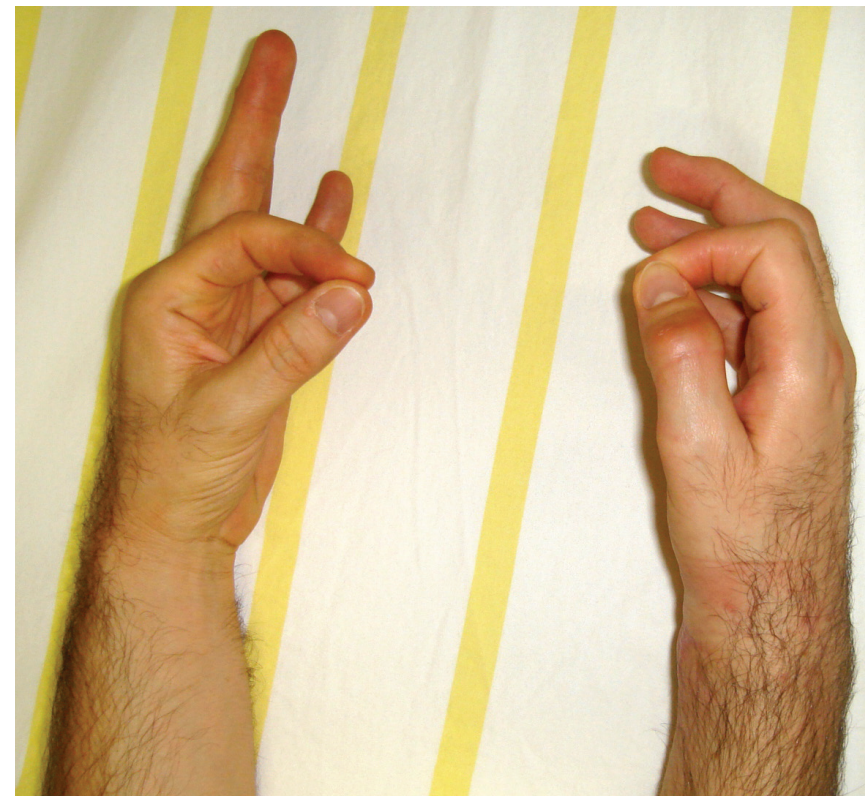

Figura 1 - Paciente apresentando traumatismo do nervo mediano no punho. Observar a garra mediana e a deficiência para movimentos de oponência do polegar.

bloqueio de condução do impulso e a recuperação se processa sem a degeneração Walleriana. É provavelmente uma lesão bioquímica. Na axonotmese (Grau II), ocorre a perda da continuidade do axônio, com preservação do tecido conjuntivo no nervo e a degeneração Walleriana. Na neurotmese, classificada em Grau III, pode haver o misto de axonotmética e neurotmética, no Grau IV, ocorre a perda de axônios, endoneuro e perineuro, mas mantém o epineuro, e no Grau $\mathrm{V}$, ocorre a secção total do nervo.

Após a desnervação, o tecido muscular sofre alterações histológicas a partir da terceira semana. A evidência clínica da atrofia e falência muscular pode ser visível em poucas semanas. Com a continuação da desnervação, principalmente com ausência do movimento, ocorre a fibrose muscular, dois a três anos após a desnervação, o músculo pode ser totalmente substituído por tecido cicatricial e gordura ${ }^{4}$.

O sucesso da regeneração neural requer ótimas condições do meio externo e interno. Relacionados à regeneração do meio interno, os fatores neurotróficos foram descritos como fundamentais na manutenção e regulação da função neuronal, incluem o NFG - fator de crescimento neural, fator de crescimento neurotrófico cerebral (BDNF), neurotrofina ${ }^{-3}$ (NT-3), neurotrofina 4/5 (NT-4/5) e neurotrofina-6 (NT-6). Embora já se conheça sobre o seu papel na função neural, estes ainda não são aplicados clinicamente ${ }^{2}$. Quanto às condições do meio externo, ressalta-se a importância do programa de reabilitação, principalmente de reeducação sensorial ${ }^{11-14}$. 


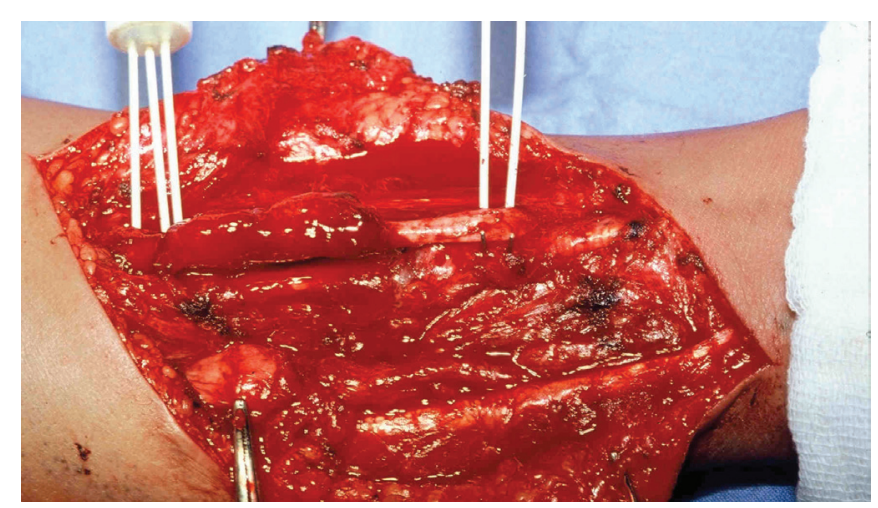

Figura 2 - Exame de potencial ativo do nervo, realizado no transoperatório em lesão de nervo mediano no antebraço com neuroma de continuidade.

\section{ESTUDO DE CONDUÇÃO DO NERVO}

Hodes et al. (1948) introduziram o método de estudo da velocidade de condução motora para avaliação das lesões de nervo periférico e hoje são aceitos para detectar a desnervação após lesão traumática do nervo ${ }^{1,2}$. Os exames utilizados para avaliação da regeneração neural são o exame clínico e eletroneuromográfico. Esses são importantes, principalmente nos casos em que as lesões foram tratadas em outros serviços e a recuperação funcional não ocorreu. De acordo com Van de Kar et al. ${ }^{16}$, a eletroneuromiografia isoladamente não fornece dados suficientes para decidir quanto à regeneração do nervo, tem valor conjuntamente com o exame físico e os achados funcionais. Os exames são realizados a cada três meses. Avalia-se a velocidade de condução motora, latência motora distal e potencial de ação (CMAP) obtido após estimulação proximal e distal (no punho e no cotovelo). A miografia é executada usando agulhas e eletrodos no abdutor curto do polegar e do dedo mínimo. Quantifica-se a densidade de desnervação, graduando-se como ausente, pouco presente ou sem desnervação e quanto ao recrutamento de fibras. No estudo retrospectivo realizado por Lester et al. ${ }^{17}$, foi verificado que a reinervação de músculos intrínsecos é anterior à resposta clínica. O nervo lesado é examinado em três semanas após o reparo, para avaliar anomalias anatômicas, e após nove meses. Se não houver sinais clínicos e eletroneuromiográficos de regeneração neural entre 9-12 meses, considerar a necessidade de reexploração cirúrgica. Se uma reexploração foi indicada, recomenda-se o uso de estimulação elétrica transoperatória na presença de neuroma de continuidade ou dúvidas quanto à integridade do nervo (Figura 2).

A incompatibilidade dos achados clínicos e eletroneuromiográficos pode ser explicada pela possibilidade de inervação cruzada, anastomose de Martin Gruber, que surge em menos de $15 \%$ da população e resulta na inervação motora anômala ${ }^{1,16,17}$.

\section{REPARO NEURAL}

\section{Neurorrafia término-terminal}

O tratamento de escolha para o reparo neural é a neurorrafia término-terminal sem tensão na linha de sutura ${ }^{1,2,18}$. Deve ser realizado logo após o trauma, preferencialmente nas primeiras seis horas. Toby et al. ${ }^{11}$ verificaram que o retardo no reparo neural de uma a duas semanas está associado a maior rigidez e, consequentemente, maior tensão na linha de sutura. Além disso, ocasionam piores resultados, maior tempo para recuperação e retorno às atividades de vida diária e trabalho.

Kline $^{1}$, Portincasa et al. ${ }^{6}$, entre outros autores ${ }^{7-10}$ descreveram o seguinte protocolo de reparo: nas lesões simples, sem contusões, contaminações, com menor perda de substância, déficit menor de $3 \mathrm{~cm}$, realizar a mobilização do tronco neural e a microsutura perineural ou epineural, com nylon 9-10/0 de acordo com o calibre do nervo; se o intervalo entre os cotos for maior de $3 \mathrm{~cm}$, utilizar enxerto autólogo fascicular ou interfascicular, de acordo com a estrutura lesada; nas lesões complexas com moderada contaminação, contusão e déficit entre os cotos menor do que $3 \mathrm{~cm}$, reavivar os cotos neurais e proceder ao reparo direto com sutura epi ou perineural; se o intervalo entre os cotos for maior do que $3 \mathrm{~cm}$, fazer a enxertia de nervo. Nos casos com extrema contaminação e tecido desvitalizado, aguardar a redução do edema e da inflamação. Algumas vezes, quando há perdas cutâneas, é necessário fazer retalhos de boa qualidade para cobertura antes do reparo neural. Além do mais, é possível, nos casos de lesões junto das articulações, o descolamento dos cotos neurais e aproximação com a flexão das articulações e imobilização gessada por quatro a oito semanas. A neurorrafia fascicular não é indicada rotineiramente, pela maior dificuldade para execução, risco de maior fibrose e desvascularização neural, e nem sempre oferecer melhores resultados. Está indicada nos casos de lesões parciais após a exploração do neuroma de continuidade. Nas lesões múltiplas de tendões e nervos, Noamam $^{5}$ utiliza o sistema de avaliação funcional em que classifica como excelente a pobre, conforme: a função tendínea, os movimentos de oposição e da musculatura intrínseca, as deformidades adquiridas e a sensibilidade. Os principais fatores de prognóstico estão relacionados à recuperação do nervo ulnar, mediano ou ambos. Os melhores resultados são vistos quando reparados primariamente, utilizando-se a epineurorrafia com microscopia e fio de nylon 9-0 ou 10-0.

\section{Enxerto de nervo}

Nos casos negligenciados, recomenda-se reparar o nervo até um ano após o trauma. Normalmente são necessários aloenxertos neurais devido à retração, grandes perdas e a distância entre os cotos neurais maior do que $2,5 \mathrm{~cm}$, que não permite o confrontamento dos cotos sem tensão na linha de sutura. Esta técnica consiste na utilização de segmento de 
nervo, mais comumente o nervo sural contra ou homolateral e o nervo cutâneo lateral do antebraço. Os resultados com o emprego de enxertos não são totalmente satisfatórios, pois a quantidade de nervos que ultrapassam as duas linhas de sutura varia entre $37-50 \%$. Vale ressaltar a importância do estudo por congelação dos cotos neurais realizado no transoperatório para avaliar a existência de fascículos viáveis nos cotos ressecados. Muito embora o uso dos enxertos seja consagrado como padrão ouro das reconstruções tardias, devido à baixa morbidade, enxertos vascularizados também são descritos, na tentativa de diminuir a cicatriz endoneural pela menor infiltração de fibroblastos ${ }^{19,20}$. Outras estruturas têm sido testadas e utilizadas para o reparo neural, como artérias, veias, músculos e materiais sintéticos, tais como tubos de silicone, gorotex e tubos bioabsorvíveis poliglicólicos. O enxerto de veia não foi aceito como alternativa até 1982, quando Chiu et al. ${ }^{21}$ apresentaram os resultados com a regeneração de fascículos e a recuperação da sensibilidade distal, funcionando como um guia e barreira ao crescimento axonal, mantendo o meio interno para regeneração ${ }^{2}$.

Os condutos de nervo podem ter um papel importante para defeitos menores, pode ser uma técnica válida, entretanto para defeitos maiores que $3 \mathrm{~cm}$ os resultados são questionáveis, embora Lundborg tenha descrito bons resultados em defeitos maiores ${ }^{2}$. Os resultados com essa técnica dependem da evolução quanto aos fatores de crescimento e cultura de células de Schwann.

A neurorrafia e a enxertia de nervo (Figuras 3 a 5) são os métodos mais comumente empregados, todavia a transferência de nervos tem ganho espaço nas reconstruções neurais, principalmente de plexo braquial. Podem ser transferidos os fascículos do nervo ulnar, mediano, espinhal acessório, sensitivo do radial, cutâneo lateral do antebraço para os músculos braquial, bíceps, supraescapular, espinhal acessório e regiões da mão, ramo motor do nervo ulnar e sensitivo para primeiro espaço, IV e V dedos, ulnar dorsal ${ }^{2}$.

\section{Neurorrafia término-lateral}

A neurorrafia término-lateral (NTL) é ainda controversa. A técnica consiste na confecção de uma janela no nervo doador sem a neurectomia ${ }^{21-23}$. Surgiu como uma alternativa para situações em que as técnicas habituais não poderiam ser usadas. Desde a primeira descrição desta técnica, em 1899, até os estudos de Viterbo, inicialmente em ratos, em 1992, esta técnica apresentava maus resultados, cujos estudos experimentais e clínicos demonstraram bons resultados, principalmente na paralisia facial e no reparo do nervo sural ${ }^{23-26}$. Para alguns autores, a aplicação desta técnica tem baixa morbidade e bons resultados, para outros, descrevem-se resultados ruins, principalmente sensitivos, acreditam que a reinervação só ocorrerá se houver a neurectomia parcial para o crescimento neural ${ }^{27-29}$. Todavia, esse crescimento no nervo

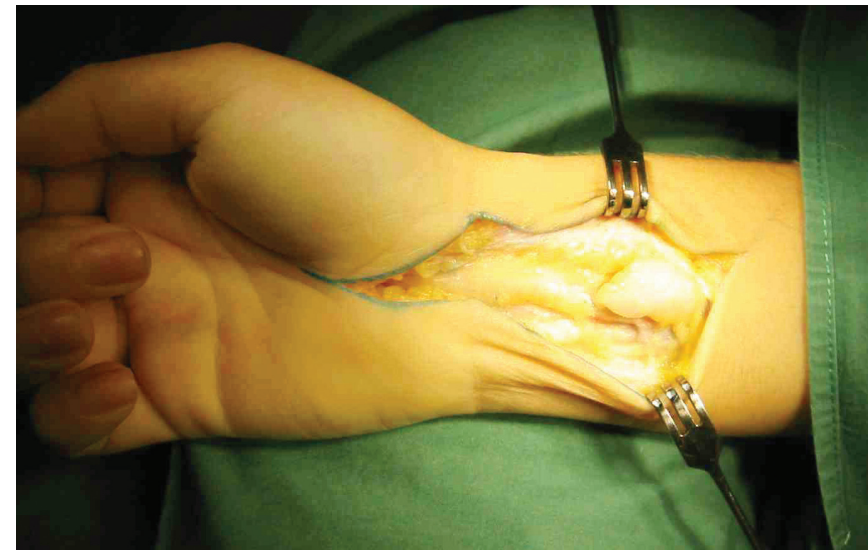

Figura 3 - Transoperatório de lesão tardia de nervo mediano. Observar o neuroma no coto proximal.

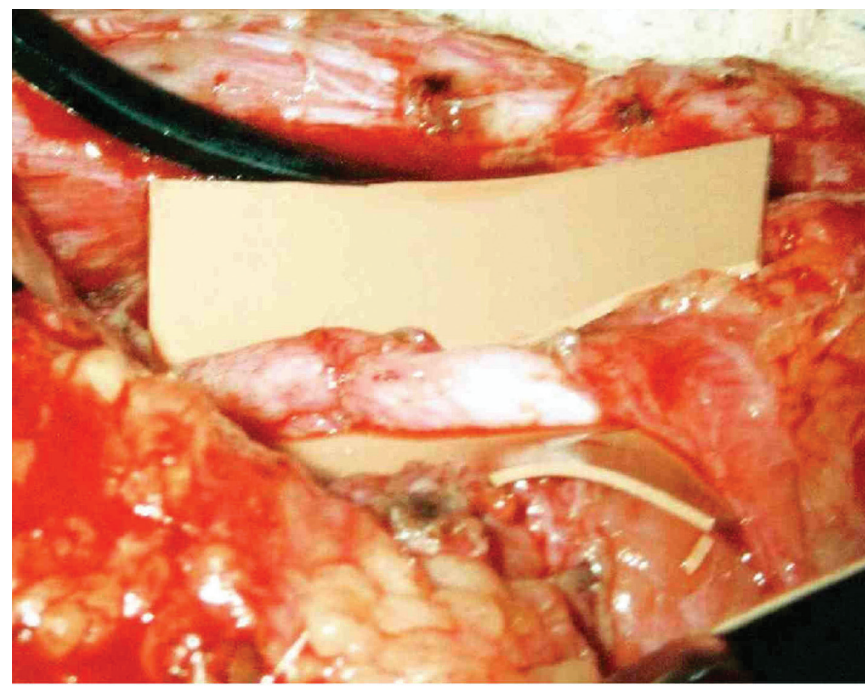

Figura 4 - Transoperatório de lesão de nervo radial após neurorrafia término-terminal com prolene 9-0.

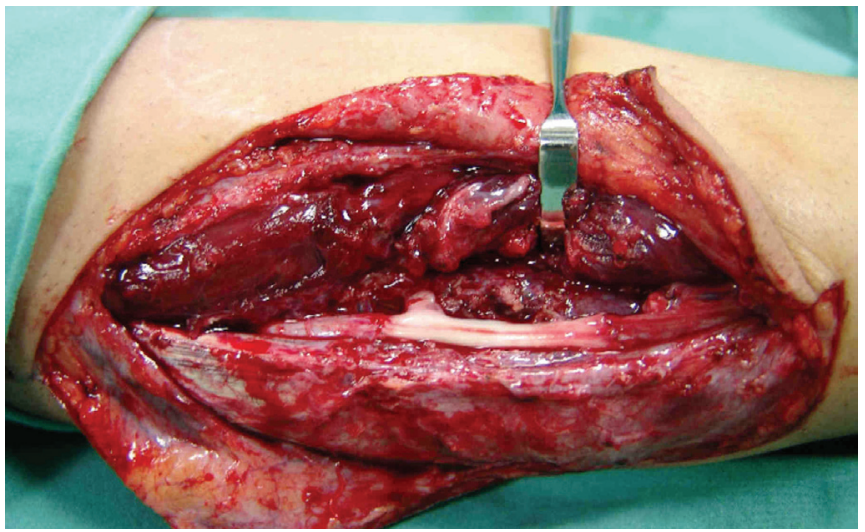

Figura 5 - Transoperatório de lesão do nervo ulnar no antebraço com interposição de dois cabos de enxerto de nervo sural e sutura com prolene 9-0. 
doador poderia ocasionar fibrose e interrupção na passagem do estímulo do nervo doador. São comumente empregadas em transferências do nervo hipoglosso para nervo facial. As aplicações clínicas da NTL ainda não foram definidas, exemplos de anastomoses mais comumente empregadas do nervo hipoglosso para o nervo facial. As publicações quanto às aplicações clínicas de NTL são relatos ou séries de casos, entre elas vale destacar a revisão de Pienaar et al. ${ }^{30}$, com 20 pacientes com lesões de plexo braquial, referindo maus resultados e abandono da técnica, e Mennen ${ }^{31}$, com 56 pacientes com lesão de nervo digital. Muitos estudos de ensaios randomizados comparando as técnicas seriam necessários para maior aplicação da neurorrafia término-lateral, pois existem vários questionamentos quanto a essa técnica, ficando a sua aplicação restrita aos casos que não há outro recurso e visando principalmente à recuperação sensitiva ${ }^{27,32}$.

\section{AVALIAÇÃO DOS RESULTADOS}

A avaliação dos resultados das lesões de nervo deve ser feita entre seis meses a dois anos, a depender do intervalo entre os cotos neurais e o momento da neurorrafia ${ }^{1,2,17}$. Testando-se a força da mão é possível avaliar o diagnóstico, resultado da cirurgia e da fisioterapia. O teste de força muscular e a escala de graduação motora do MRC (Medical Research Council, 1954) são rotineiramente utilizados (Quadro 1). São métodos rápidos e confiáveis. Entretanto, muitas das funções da mão são obtidas da musculatura intrínseca da mão, os músculos lumbricais contribuem com $32 \%$ da força de preensão. Praticamente todos os estudos avaliando a recuperação da função das lesões de nervo medem a força de preensão e pinça, existe uma dificuldade para avaliar a função da musculatura intrínseca. O teste com o dinamômetro de força e digital avalia a função dos músculos extrínsecos, extensores e intrínsecos ${ }^{1,2}$.

Jerosch-Herold ${ }^{33}$ analisou cinco testes de sensibilidade para os nervos mediano e ulnar, observou que o teste de monofilamento (WEST), locognosia ou teste de Marsh, Moberg pick-up teste mudam ao longo do tempo. São importantes na avaliação de pacientes com lesão de nervo, principalmente o teste de monofilamentos de Semmes-Weinstein Monofilament (Quadro 2). O teste de discriminação de dois pontos tem sido criticado como uma medida válida. Vale destacar que os resultados após o reparo das lesões de nervo em adultos e crianças são diferentes. Vários estudos têm demonstrado melhores resultados na criança ${ }^{1,2}$. Alguns acreditam que seja devido à melhor regeneração do nervo na criança, outros pensam que seja devido à maior plasticidade cerebral.

\section{PROGRAMA DE REABILITAÇ̃̃O PÓS-OPERATÓRIA}

O resultado, após a lesão do nervo periférico e reparação, depende de vários fatores relacionados ao local da lesão e às mudanças que ocorrem na cortical funcional ${ }^{2,34}$. O programa

\section{Quadro 1 - Graduação dos resultados pós-operatórios de lesão de nervo conforme os critérios de Louisiania State University Medical Center Grading System for Motor, usado somente para avaliação motora ${ }^{1}$.}

\begin{tabular}{l|c|c}
\hline \multicolumn{3}{c}{ Avaliação Motora } \\
\hline Grau & Evolução & Descrição \\
\hline 0 & Ausente & Sem contração muscular palpável \\
\hline 1 & Pobre & Esboço de contração muscular \\
\hline 2 & Regular & Movimento vence a gravidade e tem alguma resistência \\
\hline 4 & Moderado & Movimento vence moderada resistência \\
\hline 5 & Bom & Movimento vence o máximo de resistência \\
\hline
\end{tabular}

\section{Quadro 2 - Classificação da recuperação sensitiva conforme Semmes-Weinstein ${ }^{1}$.}

\begin{tabular}{l|l}
\hline Monofilamento & Interpretação \\
\hline $0,05 \mathrm{G}$ & Sensibilidade normal \\
$0,2 \mathrm{G}$ & Diminuição da sensibilidade discriminativa fina \\
$2,0 \mathrm{G}$ & Diminuição da sensibilidade protetora, permanecendo o suficiente \\
& para prevenir lesões. Dificuldade para discriminar forma e temperatura \\
$4,0 \mathrm{G}$ & Perda da sensibilidade protetora. Vulnerável a lesões. Perda da discriminação quente/frio \\
\hline
\end{tabular}




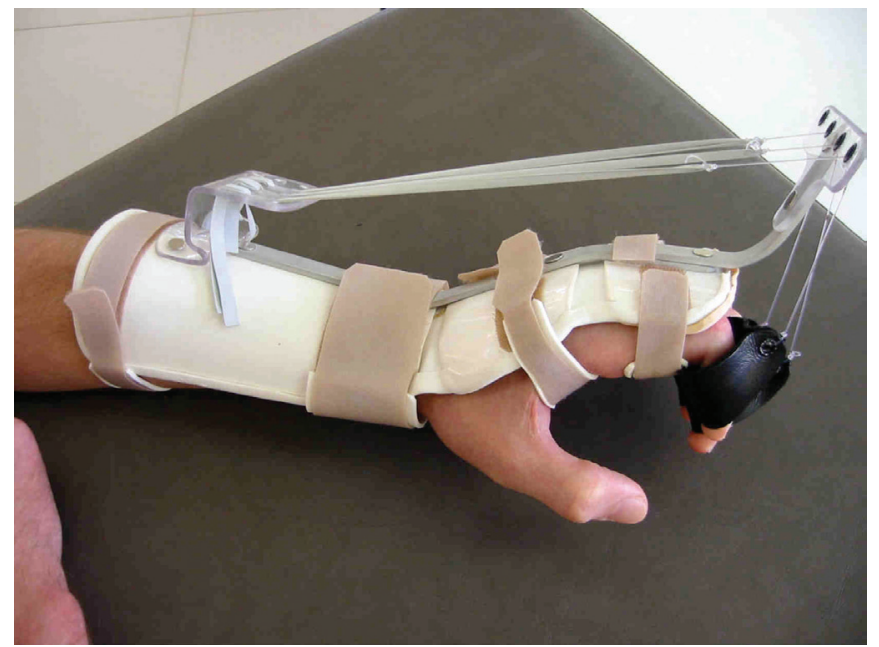

Figura 6 - Órtese dinâmica tipo Thomas para reabilitação pósoperatória do nervo radial.

de reabilitação de reaprendizagem sensorial tem demonstrado ser eficaz na recuperação das funções sensoriais. $\mathrm{Na}$ admissão realiza-se a avaliação fisico-funcional, exercícios para prevenção de estruturação de deformidades e ganho de amplitudes articulares, uso de órteses para manutenção do primeiro espaço interdigital, estabilização das articulações metacarpofalangeanas e mobilização ativa e passiva (Figura 6). O programa segue orientado por até três anos, ao longo desse prazo, reintervenções ou transferências tendíneas podem ser indicadas.

\section{CONCLUSÃO}

As lesões de nervo periférico são comuns, apesar de mais de 150 anos de experiência no reparo de nervo periférico, continua ainda como problema, principalmente nas lesões complexas de plexo braquial, com grandes perdas neurais ou abordadas muito tardiamente. Historicamente, muitos resultados descritos após reparo do nervo são pobres, o que tem inspirado pesquisas quanto a neurobiologia, neuroimunologia, pesquisas com células-tronco e condutos neurais. As técnicas de reparo com neurorrafia término-terminal epineural e a interposição de enxertos de nervo são as que apresentam os melhores resultados. Entretanto, os autores consideram como principais problemas na recuperação funcional, aqueles relacionados à técnica, à habilidade microcirúrgica, ao tempo de reparo e ao seguimento pós-operatório e programa de reabilitação.

\section{REFERÊNCIAS}

1. Kline DG. Mechanisms and pathology of injury. In: Kline DG, Hudson $\mathrm{AR}$, eds. Nerve injuries: operative results for major nerve injuries, entrapments, and tumors. Philadelphia:WB Saunders;1995.
2. Lundborg G. A 25-year perspective of peripheral nerve surgery: evolving neuroscientific concepts and clinical significance. J Hand Surg. 2000;25(3):391-414.

3. Sunderland S. Nerve injuries and their repair. A critical appraisal. Edinburgh:Churchill Livingstone; 1991.

4. Hall S. Nerve repair: a neurobiologist's view. J Hand Surg Br. 2001;26(2):129-36.

5. Noaman HH. Management and functional outcomes of combined injuries of flexor tendons, nerves, and vessels at the wrist. Microsurgery. 2007;27(6):536-43.

6. Portincasa A, Gozzo G, Parisi D, Annacontini L, Campanale A, Basso $\mathrm{G}$, et al. Microsurgical treatment of injury to peripheral nerves in upper and lower limbs: a critical review of the 8 years. Microsurgery. 2007;27(5):455-62.

7. Robinson LR. Traumatic injury to peripheral nerves. Muscle Nerve. 2000;23(6):863-73.

8. Kline DG. Civilian gunshot wounds to the brachial plexus. J Neurosurg. 1989;70(2):166-74.

9. Jaquet JB, Luijsterburg AJ, Kalmijn S, Kuypers PD, Hofman A, Horvius SE. Median, ulnar, and combined median-ulnar nerve injuries: functional outcome and return to productivity. J Trauma. 2001;51(4):687-92.

10. McAllister RM, Gilbert SE, Calder JS, Smith PJ. The epidemiology and management of upper limb peripheral nerve injuries in modern practice. J Hand Surg Br. 1996;21(1):4-13.

11. Toby EB, Meyer BM, Schwappach J, Alvine G. Changes in the structural properties of peripheral nerves after transection. J Hand Surg Am. 1996;21(6):1086-90.

12. Brushart TM, Mathur V, Sood R, Koschorke GM. Dispersion of regenerating axons across enclosed neural gaps. J Hand Surg Am. 1995;20(4):557-64.

13. Yin Q, Kemp GJ, Frostick SP. Neurotrophins, neurones and peripheral nerve regeneration. J Hand Surgery Br. 1998;23(4):433-7.

14. GelbermanRH.Nerveinjury and repair.JBone JointSurg. 2002;84B(supp III):248.

15. Wilbourn AJ. The electrodiagnostic examination with peripheral nerve injuries. Clin Plast Surg. 2003;30(2):139-54.

16. Van de Kar HJ, Jaquet JB, Meulstee J, Molenar CB, Schimsheimer RJ, Hovius SE. Clinical value of electrodiagnostic testing following repair of peripheral nerve lesions: a prospective study. J Hand Surgery Br. 2002;27(4):345-9.

17. Lester RL, Smith PJ, Mott G, McAllister RM. Intrinsic reinnervation: myth or reality? J Hand Surg Br.1993;18(4):454-60.

18. Rosén B, Dahlin LB, Lundborg G. Assessment of functional outcome after nerve repair in a longitudinal cohort. Scand J Plast Reconstr Surg Hand Surg. 2000;34(1):71-8.

19. Birch R, Raji AR. Repair of median and ulnar nerves. Primary suture is best. J Bone Joint Surg Br. 1991;73(1):154-7.

20. Kotwal PP, Gupta V. Neglected tendon and nerve injuries of the hand. Clin Orthop Relat Res. 2005;(431):66-71.

21. Chiu DT, Lovelace RE, Yu LT, Wolff M, Stengel S, Middleton L, et al. Comparative electrophysiologic evaluation of nerve grafts and autogenous vein grafts as nerve conduits: an experimental study. J Reconstr Microsurg. 1988;4(4):303-9, 311-2.

22. Viterbo F, Palhares A, Franciosi LF. Restoration of sensitivity after removal of the sural nerve: a new application of lateral-terminal neurorraphy (case report). Rev Soc Bras Cir Plast Est Reconstr. 1993;8:85-7.

23. Viterbo F. Novo método para o tratamento da paralisia facial: o "crossfacial nerve" com neurorrafia término-lateral. Rev Soc Bras Cir Plast Est Recontr. 1993;8:29-35.

24. Viterbo F. Neurorrafia término-lateral. In: Tatagiba M, Pereira CU, eds. Nervos periféricos. Tratamento clínico e cirúrgico. Rio de Janeiro: Revinter;2003. p.85-7.

25. Viterbo F, Trindade JC, Hoshino K, Mazoni Neto A. Latero-terminal neurorrhaphy without removal of epineural sheath. Experimental study in rats. Rev Paul Med. 1992;110(6):267-75. 
26. Viterbo F, Trindade JC, Hoshino K, Mazzoni Neto A. End-to-side neurorrhaphy with removal of the epineural sheath: an experimental study in rats. Plast Reconstr Surg. 1994;94(7):1038-47.

27. Flores LP. Neurorrafia término-lateral: uma evolução da técnica de reparo de lesões de nervos periféricos? Arq Bras Neurocir. 2006;25(2):66-73.

28. Kayikçioglu A, Karamürsel S, Agaoglu G, Keçik A, Celiker R, Cetin A. End-to-side neurorrhaphies of the ulnar and median nerves at the wrist: report of two cases without sensory or motor improvement. Ann Plast Surg. 2000;45(6):641-3.

29. Al-Qattan MM. Terminolateral neurorrhaphy: review of experimental and clinical studies. J Reconstr Microsurg. 2001;17(2):99-108.
30. Pieenar C, Swan MC, De Jager W, Solomons M. Clinical experience with end-to-side nerve transfer. J Hand Surg Br. 2004;29(5):438-43.

31. Mennen U. End-to side nerve suture in clinical pratice. Hand Surg. 2003;8(1):33-42.

32. Sanapanich K, Morrison WA, Messina A. Physiologic and morphologic aspects of nerve regeneration after end-to-end or end-to-side coaptation in a rat model of brachial plexus injury. J Hand Surg Am. 2002;27(1):133-42.

33. Jerosch-Herold C. Measuring outcome in median nerve injuries. J Hand Surg Br. 1993;18(5):624-8.

34. Rosén B, Lundborg G. Sensory re-education after nerve repair: aspects of timing. Handchir Mikrochir Plast Chir. 2004;36(1):8-12.

Correspondência para:

Katia Torres Batista

SMHS Qd 501, cjto A - Brasília, DF, Brasil - CEP 70335-901

E-mail: katiatb@terra.com.br 